\title{
Graduated Scale Inspection Using Computer Vision
}

\author{
Benjamín Valera, Sergio Padilla, Gabriel Chávez, and Victor García Garduño \\ Centro de Ciencias Aplicadas y Desarrollo Tecnológico, UNAM, Ciudad Universitaria, \\ 04510 D.F., México \\ metro@aleph.cinstrum.unam.mx
}

\begin{abstract}
A method for graduated scale inspection using computer vision is proposed. We deal mainly with the lens distortion problem in the image acquire device due to its influence in the uncertainty of the graduated scale inspection process. This paper presents an algorithm for image correction by means of camera calibration and distortion compensation. The camera calibration method provides the ideal undistorted coordinates of the system using as input distorted images of a $2 \mathrm{D}$ calibration pattern. The distortion compensation stage is implemented using the ideal undistorted coordinates as an unwarped mesh. Then distortion compensation can be applied to any image acquired with the system, improving the inspection procedure. Test results using real data are presented. Also, we describe the image feature extraction approach used in order to automate the process.
\end{abstract}

\section{Introduction}

There is an important effort to take advantage of computer vision systems in the dimensional metrology field [1]. Computer vision hardware and software have been used to improve calibration, measurement or inspection process. However, main problems arise when high accuracy is needed in order to accomplish with high-quality uncertainty levels. This is the case in the graduated scale inspection process, where the uncertainty source comes from lens distortion in the image intensifier device coupled to the image acquisition system. Consequently, lens distortion must be taken into account in measurements or inspections outcomes.

The graduated scale inspection process consists of a comparison approach between the scale under inspection and a length standard, as Fig.1 shows.

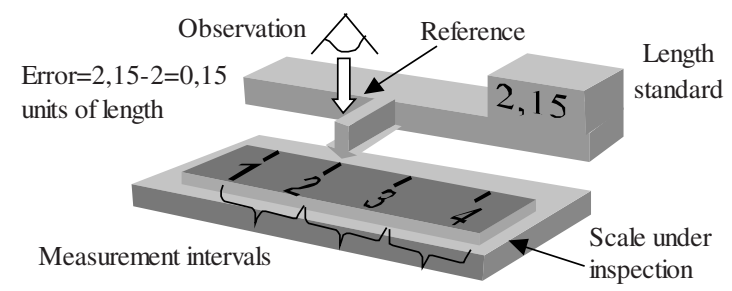

Fig. 1. Scale graduated inspection. A typical scale is compared against a length standard. 
In Fig. 1 the reference cursor on the length standard is placed mechanically against a measurement interval on the scale under inspection. The user does this through an observation device. For this example the measurement error is calculated as the difference in the length standard, 2,15, and the scale under inspection, 2,0, resulting in an error of 0,15 units of length. In our practical implementation of the layout in Fig. 1, we use an optical encoder as the length standard, a multimedia camera with microscope as the observation device and a mechanical artifact that supports the whole arrangement in concordance with international recommendations [4]. Then the accuracy of the inspection resides in the exactness of the positioning of the cursor over the printed graduation in the scale. Two factors have influence in the positioning: the mechanical device and the good judgment of the user that is controlling the inspection through the observation device. We pay attention in the second factor; supplying undistorted images to the user, thus improving the visual inspection.

This paper then deals with image distortion correction for the particular case of graduated scale inspection using the layout described. In particular, the distortion generated by the combination of the camera and microscope lenses, provides deformations that greatly fails with the classical distortion models described in the literature [6]. Instead, we adopt a mesh unwarping approach in which the whole image is subdividing and a local correction is applied to each independent region. The camera calibration procedure [2] provides a reference unwarped mesh, which takes as input real distorted images of a $2 \mathrm{D}$ grid pattern and a least squares approach leads to a "straight lines in the scene should be straight in the image" sense. Furthermore, image feature extraction is used in order to achieve a reliability system and automation.

The paper is organized as follows. Section 2 describes the camera calibration method by using real distorted images of a $2 \mathrm{D}$ calibration pattern. A warped mesh is obtained by means of image feature extraction and it is used by the least squares approach to derive the unwarped mesh. Section 3 presents the distortion compensation algorithm. The algorithm takes both warped and unwarped meshes to reproduce unwarped images. The performance of our method is demonstrated in section 4 by the results of applying distortion compensation to real images of graduated scales. Section 5 gives concluding remarks and future work.

\section{Camera Calibration Method}

In the camera calibration procedure, we use an approach similar to the pinhole camera model [5]. In the model presented in this paper there are two main differences. The first is that the scene is magnified and not inverted compared to the pinhole camera [2]. In the second, the short field-of-view of the acquisition system imposes planar scenes with no depth. Then a $2 \mathrm{D}$ calibration pattern is used and the depth remains as a free parameter. The calibration model is shown in Fig. 2. In this figure, the calibration pattern is placed in the target plane, $T$, and the image is formed in the image plane, $I$. The model is completed with the focal plane, $F$, which contains de focal point. Then, a feature in the calibration pattern, $M$, is projected to the image plane, $m$. Focal and image planes are separated $f$ units and target and image plane are at $d$ units of length. The image plane has two components: distorted and undistorted, both related by a distortion model. In order to perform image-processing tasks, image coordinates are 
stored and managed in row-column computer arrays. The distortion produces warped and unwarped computer arrays. The parallel depth alignment ( $Z$ axis) between the target and image planes is achieved by focusing.

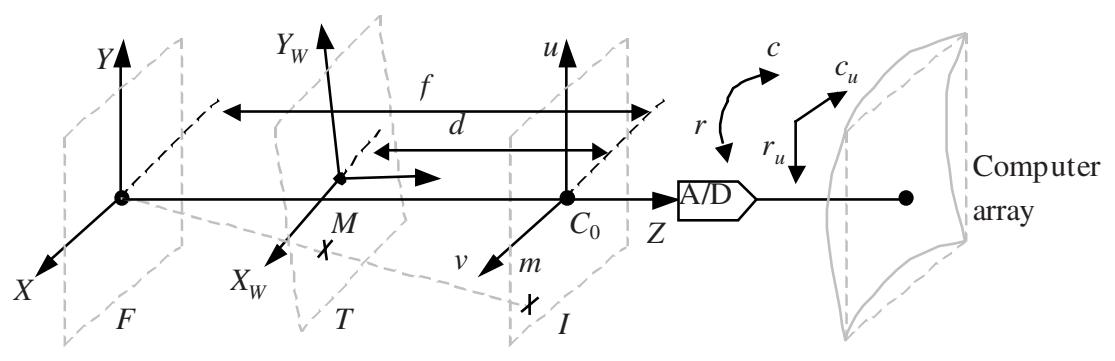

Fig. 2. Camera model. A geometrical model relates global coordinate to array coordinates.

The main idea of calibration is to obtain a set of unwarped features starting from a set of warped features. As we will show, features are line crossings in the image formed by the calibration target. To achieve automation, the procedure is performed in two stages: feature extraction and distortion estimation.

\subsection{Feature Extraction}

Feature extraction is performed with some modifications as described in [2]. The procedure starts with an original image of a $0,5 \mathrm{X} 0,5 \mathrm{~mm}$ calibration grid (Fig. 3(a)). Then, a gray scale closing filter extracts the background. The subtraction between the original image and its background followed by thresholding yields to a simplified image. Horizontal and vertical line extraction is applied to the binary image by convolving with horizontal and vertical structural elements. The feature image in Fig. 3(b) is obtained by computing the center of gravity of the horizontal and vertical intersection. Each center of gravity conforms the starting point in the search of a finest subpixel feature extraction.

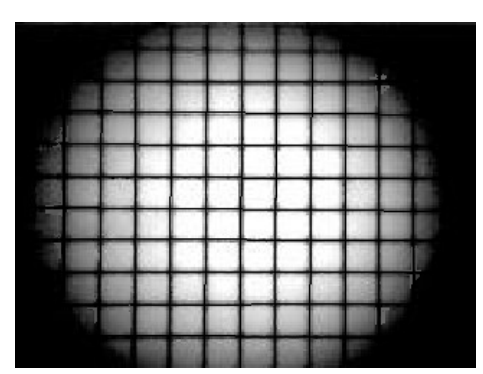

(a)

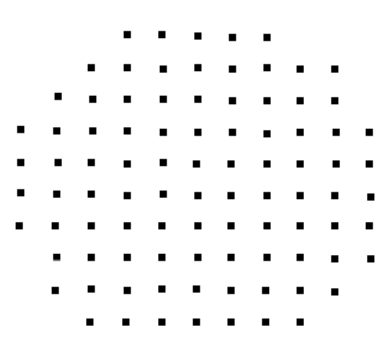

(b)

Fig. 3. Image pre-processing using morphological. 
The crossing points of the vertical and horizontal lines are extracted with subpixel accuracy through a method based on the starting point and the analysis of a squared pattern around it, similar to the one proposed in [3]. This method avoids the iterative minimization procedure described in [2] and constitutes a closed form solution in order to gather computation speed without a loose of exactness. The main idea surrounding the algorithm is to fit lines to image data and then found the intersection point. Two lines are needed for subpixel feature extraction: horizontal and vertical. The lines are defined by analyzing the original grayscale image along a squared pattern centered at starting points. The Fig. 4 shows a detail of the sampled squared pattern around the starting point. The gray level profile of the pattern defines eight border points marked as circles signs in Fig. 4(a). The border points are arranged in horizontal and vertical pairs to find their midpoints. Then the four midpoints, marked as triangles signs in Fig. 4(a), define the two lines. Simple mathematics is used to locate the intersection point with subpixel accuracy marked with a star sign. The Fig. 4(b) shows a typical gray level profile of the squared pattern. The first order derivative of the profile is computed. Then, the local maxima of derivative is detected and grouped into pairs to conform the border points. Since the original image is on a discrete domain, the gray level intensity is computed using bilinear interpolation.

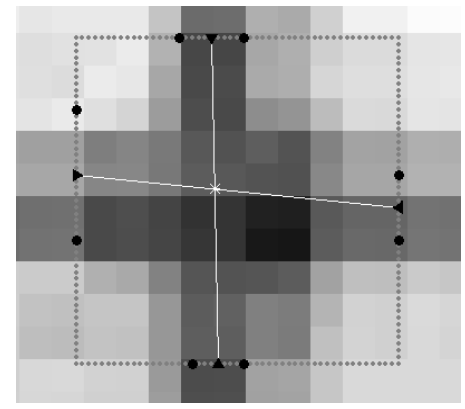

(a)

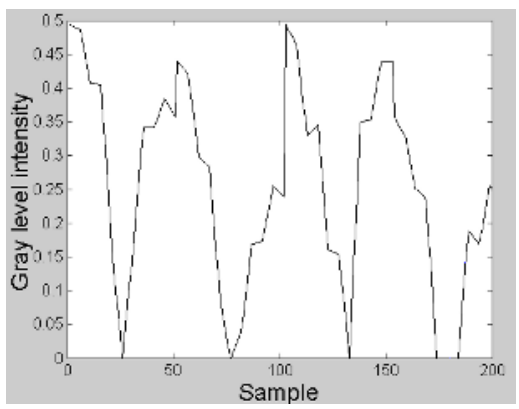

(b)

Fig. 4. Squared pattern of the original grayscale image and its samples.

Then the whole image is processed using the method described in order to extract the $N$ entire feature points. The Fig. 5(a) shows the extracted points overlying the original image. Additional processing is required to correlate warped feature points, $\left(r_{i}, c_{i}\right)$, against their global position defined in the grid pattern, $\left(X_{w i}, Y_{w i}\right)$, where $i=1$, $\ldots, N$ points. First, horizontal and vertical images are labeling with a consecutive number starting from one. Then, 2D global positions are assigned to feature points by searching its position in both labels image. The $X_{w i}$ and $Y_{w i}$ coordinates are calculated through multiplying the size of the grid and the label number. Fig. 5(b) shows the 2D world coordinate assignment. 


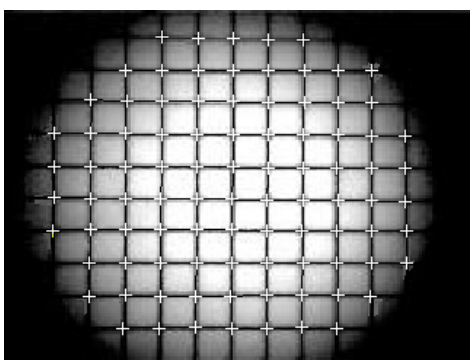

(a)

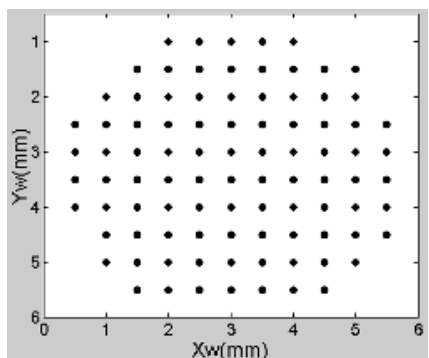

(b)

Fig. 5. Feature points with subpixel accuracy and their correlation with $2 \mathrm{D}$ global positions.

\subsection{Distortion Estimation}

Distortion estimation is performed by first generating an unwarped set of points from the extracted warped features, their correlation with $2 \mathrm{D}$ global positions and the camera calibration model. In the camera calibration model, the main idea is to obtain a set of parameters that relates world positions, $\left(X_{w i}, Y_{w i}\right)$ to unwarped computer array, $\left(r_{u i}, c_{u i}\right)$. Following the procedure described in [2] this relation is

$$
\left[\begin{array}{c}
c_{u} \\
r_{u}
\end{array}\right]=\left[\begin{array}{ccc}
a_{u} & -a_{v} & t_{u} \\
a_{v} & a_{u} & t_{v}
\end{array}\right]\left[\begin{array}{c}
X_{w} \\
Y_{w} \\
1
\end{array}\right]
$$

where

$$
a_{u}=s \cos \theta a_{v}=s \sin \theta t_{u}=s t_{x}+c_{0} t_{v}=s t_{y}+r_{0} s=\frac{f s_{u}}{Z_{w}+f-d}=\frac{f s_{v}}{Z_{w}+f-d}
$$

and $t_{x}, t_{y}$ are translation in $X$ and $Y, \theta$ is the rotation in the $Z$ direction, $\left(r_{0}, c_{0}\right)$ is the pixel position of the origin $C_{0}$, and $s_{u}=s_{v}$ is the aspect factor of the ccd camera.

Then the distortion estimation is derived as the relationship between unwarped, $\left(r_{u i}\right.$, $\left.c_{u i}\right)$, and warped, $\left(r_{i}, c_{i}\right)$ computer arrays. The equation (1) should fit a set of $N$ observations $\left(c_{i}, r_{i}\right),\left(X_{w i}, Y_{w i}\right) i=1, \ldots, N$ to the model in the equation with adjustable parameters $\rho=\left(a_{u}, a_{v}, t_{u}, t_{v}\right)$. Therefore, $\rho$ should minimize the following error

$$
\gamma(\boldsymbol{\rho})=\frac{1}{N} \sum_{i=1}^{N}\left[\left(c_{i}-c_{u i}\right)^{2}+\left(r_{i}-r_{u i}\right)^{2}\right]
$$

Substituting equation (1) into (2) and taken into account that at the minimum the partial derivatives of $\gamma$ with respect to $\rho$ vanish, we obtain the following 


$$
\left[\begin{array}{cccc}
C & 0 & A & B \\
0 & C & -B & A \\
A & -B & 1 & 0 \\
B & A & 0 & 1
\end{array}\right]\left[\begin{array}{l}
a_{u} \\
a_{v} \\
t_{u} \\
t_{v}
\end{array}\right]=\left[\begin{array}{c}
D_{1} \\
D_{2} \\
C_{0} \\
R_{c}
\end{array}\right]
$$

The equation (3) can be resolved for $\rho$ taken as input data 2D global positions $\left(X_{w i}\right.$, $\left.Y_{w i}\right)$ and their correlated projections into the image plane $\left(c_{i}, r_{i}\right)$, since

$$
\begin{gathered}
A=\frac{1}{N} \sum_{i=1}^{N} X_{w i} \quad B=\frac{1}{N} \sum_{i=1}^{N} Y_{w i} \quad C=\frac{1}{N} \sum_{i=1}^{N}\left(X_{w i}^{2}+Y_{w i}^{2}\right) \\
R_{c}=\frac{1}{N} \sum_{i=1}^{N} r_{i} C_{0}=\frac{1}{N} \sum_{i=1}^{N} c_{i} D_{1}=\frac{1}{N} \sum_{i=1}^{N}\left(c_{i} X_{w i}+r_{i} Y_{w i}\right) D_{2}=\frac{1}{N} \sum_{i=1}^{N}\left(r_{i} X_{w i}-c_{i} Y_{w i}\right)
\end{gathered}
$$

Then, equation (1) can be used to generate an unwarped grid using the warped features and their correlation with 2D global positions (Fig. 6).

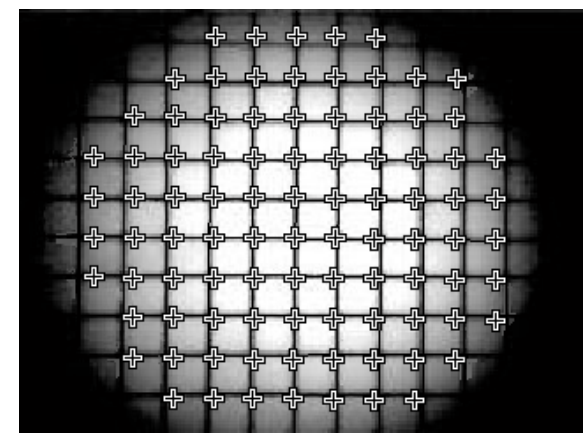

Fig. 6. Unwarped grid overlying the original warped image.

\section{Distortion Compensation Algorithm}

We test with the following well-known model of distortion

$$
r_{u}=r+\delta_{r}(r, c) \quad c_{u}=c+\delta_{c}(r, c)
$$

where $r_{u}$ and $c_{u}$ are the ideal unwarped coordinates, $r$ and $c$ are the corresponding warped coordinates and $\delta_{r}(r, c)$ and $\delta_{c}(r, c)$ are bi-variable polynomials of $n$ degree. The bi-variable polynomial method fails in representing the real system distortion. This is due to the fact that local distortions are not in agreement with the proposed model. In general, cheap lenses, like the one used in this vision system, presents a substantial amount of local distortion. Therefore, to increase the accuracy in the distortion compensation stage, we introduce a region-based approach similar to the mesh-warping algorithm used in image morphing [7]. This algorithm requires both source and destination meshes to perform bilinear transformations between regions 
inside the mesh. As a first steep and referring to the Fig. 7(a), a uniform auxiliary mesh (circle sign) is placed on top the non-uniform mesh (dot sign) obtained in the feature extraction stage. In this sense, there are defined quadrilateral regions and the features that do not define quadrilaterals are discarded. Quadrilateral regions are marked with the triangle sign, which is enclosed by only four feature points marked with both circle and dot signs. Then, warped quadrilateral sub-regions are transformed to the corresponding quadrilateral unwarped sub-region as shown in Fig. 7(b), according with the following backward transformation formula

$$
r=a_{0}+a_{1} r_{u}+a_{2} c_{u}+a_{3} r_{u} c_{u} \quad c=b_{0}+b_{1} r_{u}+b_{2} c_{u}+b_{3} r_{u} c_{u}
$$

where parameters $a$ and $b$ are defined by the knowledge of the eight vertex points as follows

$$
\left[\begin{array}{c}
r_{0} \\
r_{1} \\
r_{2} \\
r_{3}
\end{array}\right]=\left[\begin{array}{cccc}
1 & r_{u 0} & c_{u 0} & r_{u 0} c_{u 0} \\
1 & r_{u 1} & c_{u 1} & r_{u 1} c_{u 1} \\
1 & r_{u 2} & c_{u 2} & r_{u 2} c_{u 2} \\
1 & r_{u 3} & c_{u 3} & r_{u 3} c_{u 3}
\end{array}\right]\left[\begin{array}{l}
a_{0} \\
a_{1} \\
a_{2} \\
a_{3}
\end{array}\right]\left[\begin{array}{l}
c_{0} \\
c_{1} \\
c_{2} \\
c_{3}
\end{array}\right]=\left[\begin{array}{cccc}
1 & r_{u 0} & c_{u 0} & r_{u 0} c_{u 0} \\
1 & r_{u 1} & c_{u 1} & r_{u 1} c_{u 1} \\
1 & r_{u 2} & c_{u 2} & r_{u 2} c_{u 2} \\
1 & r_{u 3} & c_{u 3} & r_{u 3} c_{u 3}
\end{array}\right]\left[\begin{array}{l}
b_{0} \\
b_{1} \\
b_{2} \\
b_{3}
\end{array}\right]
$$

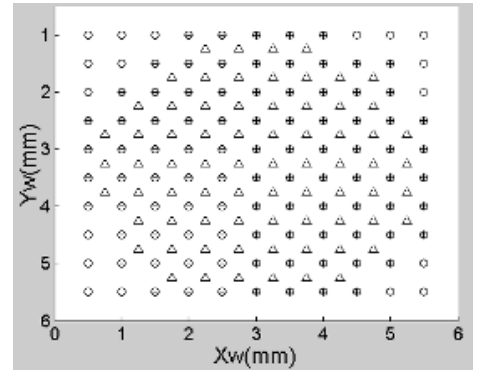

(a)

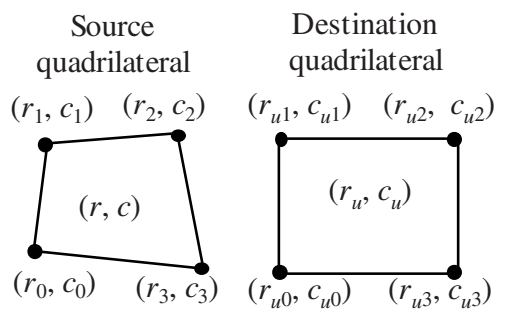

(b)

Fig. 7. Region-based approach for distortion compensation.

Finally, applying the backward transformation in (4) to the whole original image, we obtain the unwarped image shown in Fig 8(a). Once the camera is calibrated using the procedure described here, the visual inspection of a graduated scale can be improved with the backward transformation as shown in Fig 8(b).

\section{Results}

In order to obtain quantitative results, we perform two experiments to proof the "straight lines in the scene should be straight in the image" sense. In the first experiment, we fit the unwarped feature points to straight lines and the squared sum of the residuals is compared against the one obtained with warped features. The 


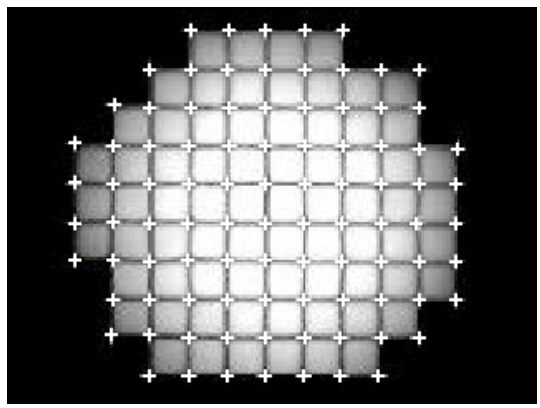

(a)

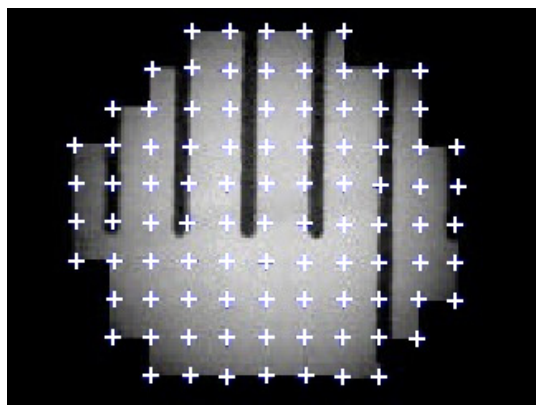

(b)

Fig. 8. Distortion compensation using the region-based approach described.

squared sum of residuals for the warped grid is 19,4188 (pixels) against $1,4086 \times 10^{-15}$ for the unwarped one. In the second experiment we test the uniformity in distance between vertical and horizontal lines in the warped and unwarped images of the calibration grid. We obtained a uniform distance between lines of 23,2 pixels for the unwarped case against the non-uniform distance of 21,6 to 25 pixels for the warped case. Then, in our mind, the two experiments are indicatives of the main idea shaped to project the scene without distortion. Further results of automated feature extraction, camera calibration and distortion compensation procedures were presented all along the paper.

\section{Conclusions and Future Work}

A computer vision system for graduated scale inspection was presented. The main problem faced up was the optical distortion in the acquisition hardware. The generation of a reference unwarped set of features was done using camera calibration tools. Then the algorithm generates nearly ideal images by the distortion compensation method. Also, feature extraction and image processing achieved the automation of the process. The results indicate us the reliability of the approach in the "straight lines in the scene should be straight in the image" sense. For metrological purposes, the accuracy of the system was tested qualitatively and quantitatively by the development of two experiments.

In the future, additionally image processing can be done in order to complete the automation of the measurement process and avoid the human intervention. In our idea, the layout presented in the Fig. 1 will suffer of few changes. First, the length standard will be precisely placed in a known position. Then, the vision system will assign a measurement to the observation process. Then, the main problem is concerned with the human knowledge that is used in a measurement with graduated scales. 
Acknowledgments. This work has been financially supported by DGAPA-UNAM. The authors of this paper are indebt to the grant No IN111001 from PAPIIT.

\section{References}

1. Aguilar, J. J., Torres, F. and Lope, M. A.: Stereo Vision for 3D Measurement: Accuracy Analysis, Calibration and Industrial Applications. Measurement 184 (1996) 193-200.

2. Cañero, C., Vilariño, F., Mauri, J. and Radeva, P.: Predictive (un)distortion model and 3D Reconstruction by Biplane Snakes. IEEE Transactions on Medical Imaging 219 (2002) 1188-1201.

3. Harris, K., Efstratiadis, S. N., Maglaveras, N., Gourassas, J., Pappas, C. and Louridas, G.: Coronary Arterial Tree Extraction based on Artery Tracking and Mathematical Morphology. Computers in Cardiology (1988) 769-772.

4. International Recommendation No. 35: Material Measures of length for General Use. OIML (1985) 22.

5. Tsai, R. Y.: A Versatile Camera Calibration Technique for High-Accuracy 3D Machine Vision Metrology Using Off-the-Shelf TV Cameras and Lenses. IEEE Journal of Robotics and Automation 34 (1987) 323-344.

6. Weng, J., Cohen, P. and Herniou, M.: Camera Calibration with Distortion Models and Accuracy Evaluation. IEEE Transactions on Pattern Analysis and Machine Intelligence 14 10 (1992) 965-980.

7. Woldberg, G.: Digital Image Warping. Wiley (2002) 340. 